\title{
Programmed death ligand 1 (PD-L1) in colon cancer and its interaction with budding and tumor-infiltrating lymphocytes (TILs) as tumor-host antagonists
}

\author{
Corinna Lang-Schwarz ${ }^{1,3}$ (1) $\cdot$ Balint Melcher ${ }^{2} \cdot$ Arndt Hartmann $^{3} \cdot$ Simone Bertz $^{3} \cdot$ Theresa Dregelies $^{1}$. \\ Klaus Lang-Schwarz ${ }^{4}$ Michael Vieth ${ }^{1,3} \cdot$ William Sterlacci $^{1,3}$
}

Accepted: 16 June 2021 / Published online: 25 June 2021

(c) The Author(s) 2021

\begin{abstract}
Purpose To analyze the role of programmed death ligand 1 (PD-L1) immunohistochemisty in the context of tumor microenvironment in colon cancer (CC) with focus on the interaction between tumor budding and tumor-infiltrating lymphocytes (TILs) and to elucidate its potential value for immunooncologic treatment decisions.

Methods Three hundred forty seven patients with CC, stages I to IV, were enrolled. PD-L1 immunohistochemistry was performed using two different antibodies (clone 22C3 pharmDx, Agilent and clone QR1, Quartett). Tumor proportion score (TPS) as well as immune cell score (IC) was assessed. Budding and TILs were assessed according to the criteria of the International Tumor Budding Consensus Conference (ITBCC) and International TILs Working Group (ITWG). Correlation analyses as well as survival analyses were performed.

Results PD-L1 positivity significantly correlated with TILs > 5\% and MMR deficiency, and PD-L1-positive cases (overall and IC) showed significantly longer overall survival (OS) with both antibodies.The parameters "high grade," "right-sidedness," and "TILS > 5\% regardless of MMR status" evolved as potential parameters for additional immunological treatment decisions. Additionally, TPS positivity correlated with low budding. More PD-L1-positive cases were seen in both high TIL groups. The low budding/high TIL group showed longer disease-free survival and longer OS in PD-L1-positive cases.

Conclusion Overall, PD-L1 positivity correlated with markers of good prognosis. PD-L1 immunohistochemistry was able to identify parameters as additional potential candidates for immune therapy. Furthermore, it was able to stratify patients within the low budding/high TIL group with significant prognostic impact.
\end{abstract}

Keywords Budding $\cdot$ Tumor-infiltrating lymphocytes (TILs) $\cdot$ Colon cancer $\cdot$ PD-L1

\section{Introduction}

Colorectal cancer (CRC) is one of the most common cancer types worldwide. In 2020, over 1.9 million people were newly diagnosed with CRC and about 935,000 people died from CRC [1].

Corinna Lang-Schwarz

Corinna.Lang-Schwarz@klinikum-bayreuth.de

1 Institute of Pathology, Klinikum Bayreuth, Preuschwitzer Str. 101, 95445 Bayreuth, Germany

2 Institute of Pathology, Koblenz, Franz-Weis-Str. 13, 56073 Koblenz, Germany

3 Institute of Pathology, Friedrich-Alexander-University, Erlangen-Nuremberg, Krankenhausstr. 8-10, 91054 Erlangen, Germany

4 Department of Anesthesiology, Klinikum Bayreuth, Preuschwitzer Str. 101, 95445 Bayreuth, Germany
Until recently, treatment regimens were based on the tumor node metastasis (TNM) staging system, the grading according to the World Health Organization (WHO) classification and molecular biomarkers [2]. The discovery of immune checkpoint inhibitors has revolutionized cancer treatment regimes, and checkpoint inhibitors have already become part of the therapeutic standard in different human cancer types, for example (but not limited to), lung cancer, malignant melanoma, and breast cancer [3-6].

In mismatch repair-deficient (dMMR) metastatic colon cancer (CC), the progammed death 1 (PD-1) inhibitor pembrolizumab led to a significantly longer progression-free survival than chemotherapy when applied as first-line therapy and showed fewer treatment-related adverse events [7]. Therefore, in June 2020, the US Food and Drug Administration (FDA) approved pembrolizumab (KEYTRUDA, Merck Sharp Dohme) for the first-line treatment of patients with 
unresectable or metastatic microsatellite instability-high (MSI-H) or dMMR CC, independent from PD-L1 immunohistochemisty [8]. Infact, mismatch repair status seems to be the only reliable feature in $\mathrm{CC}$ to predict treatment response to checkpoint inhibitor therapy. So far, studies failed to prove the predictive value of PD-L1 immunohistochemistry in CC.

However, the group of MSI-H or dMMR CC counts for only up to $15 \%$ of all patients with CRC, only about $5 \%$ of them being stage IV [9-13].

Additionally, in recent years, additive features with focus on tumor microenvironment have gained increasing attention as they have shown potential to predict prognosis or response to therapy or even serve as therapeutic targets.

Among them, on the tumor side, tumor budding, as a morphologic sign of epithelial-mesenchymal transition (EMT), is associated with higher tumor stage (T) and higher nodal status $(\mathrm{N})$, venous invasion (V1) and lymphatic vessel infiltration (L1), local tumor recurrence, distant metastasis, and higher tumor agressiveness [14-25]. It is now accepted as an additional prognostic factor for CRC, according to the Union for International Cancer Control (UICC), and listed among the essential and desirable diagnostic criteria for CRC in the current fifth edition of WHO Classification of Tumours [2, 26].

On the host immunity side, tumor-infiltrating lymphocytes (TILs) are also a popular research object in various cancer types. Increased TILs in CRC are an independent predictor of better prognosis [22, 27, 28]. Assessment and reporting of budding as well as TILs on hematoxylin and eosin (H\&E) stained slides have recently been well defined and validated by international groups [29-32]. We could previously show that the combination of tumor budding and TILs as tumor-host antagonists is able to further stratify patients with $\mathrm{CC}$ regarding overall survival (OS) and to identify patients in stage II and III CC regarding the benefit from adjuvant chemotherapy $[22,25,33]$.

Therefore, the aim of our study was the following:

- To analyze the immunohistochemical PD-L1 staining pattern in a large series of CC, stages I-IV.

- To elucidate the role of PD-L1 immunohistochemistry in the context of the "budding and TIL combination" as tumor-host antagonists.

- To identify PD-L1-positive "budding/TIL" subgroups which might qualify as potential additional candidates for future immunooncogenic treatment decisions.

\section{Methods}

\section{Case selection}

The study cohort consisted of 347 cases of CC, stages I to IV, diagnosed at the Institute of Pathology, Klinikum Bayreuth GmbH, Bayreuth, Germany between 2005 and 2016.
Cases with neoadjuvant treatment modalities and rectal carcinoma (due to high percentage of neoadjuvant treatment) were excluded. Further patient and tumor characteristics are listed in Table 1.

Table 1 Summary of patient and tumor characteristics

\begin{tabular}{|c|c|}
\hline Feature & Frequency, $n(\%)$ \\
\hline Age (year; mean, $\max , \min , n=347$ ) & 75 (47-97) \\
\hline \multicolumn{2}{|l|}{ Gender $(n=347)$} \\
\hline Male & $161(46.4)$ \\
\hline Female & $186(53.6)$ \\
\hline \multicolumn{2}{|l|}{$\mathrm{pT}(n=347)$} \\
\hline pT1 & $23(6.6)$ \\
\hline pT2 & $39(11.2)$ \\
\hline pT3 & $206(59.4)$ \\
\hline pT4 & $79(22.8)$ \\
\hline $\mathrm{pN}(n=347)$ & $198(57.1)$ \\
\hline pNO & $82(23.6)$ \\
\hline $\begin{array}{l}\mathrm{pN} 1 \\
\mathrm{pN} 2\end{array}$ & $67(19.3)$ \\
\hline \multicolumn{2}{|l|}{$\mathrm{M}(n=347)$} \\
\hline M0 & $292(84.1)$ \\
\hline M1 & $55(15.9)$ \\
\hline \multicolumn{2}{|l|}{ TNM stage $(n=347)$} \\
\hline I & $51(14.7)$ \\
\hline II & $140(40.3)$ \\
\hline III & $97(28.0)$ \\
\hline IV & $59(17.0)$ \\
\hline \multicolumn{2}{|l|}{$\begin{array}{l}\text { Tumor location } \\
\text { (right/left, } n=347 \text { ) }\end{array}$} \\
\hline Right & $238(68.6)$ \\
\hline Left & $109(31.4)$ \\
\hline \multicolumn{2}{|l|}{ Grading (WHO 2019, $n=347$ ) } \\
\hline Low grade & $277(79.8)$ \\
\hline High grade & $70(20.2)$ \\
\hline \multicolumn{2}{|l|}{ Venous invasion $(n=347)$} \\
\hline V0 & $274(79.0)$ \\
\hline $\mathrm{V} 1$ & $73(21.0)$ \\
\hline \multicolumn{2}{|l|}{ Lymphatic invasion $(n=347)$} \\
\hline L0 & $211(60.8)$ \\
\hline L1 & $136(39.2)$ \\
\hline \multicolumn{2}{|l|}{ Mucinous (y/n; $n=347)$} \\
\hline y & $24(6.9)$ \\
\hline n (NOS) & $323(93.1)$ \\
\hline \multicolumn{2}{|l|}{ MMR status $(n=312)$} \\
\hline MMR proficient & $239(76.6)$ \\
\hline MMR deficient & $73(23.2)$ \\
\hline \multicolumn{2}{|l|}{$\operatorname{KRAS}(n=93)$} \\
\hline Wild type & $49(52.7)$ \\
\hline Mutated & $44(47.3)$ \\
\hline
\end{tabular}

TNM tumor node metastasis, WHO World Health Organization, NOS not otherwise specified, MMR mismatch repair, KRAS Kirsten rat sarcoma 
Follow-up data were provided from the local tumor registry in Bayreuth. A complete follow-up was available for 308 cases. Median follow-up was 30 months (range 0-137 months). One hundred ninety-seven patients were alive at study end; 111 died.

The ethics commission of Friedrich-Alexander-University Erlangen-Nuremberg approved the study (study number 216_19 $\mathrm{Bc})$.

\section{Histological assessment of budding and TILs}

H\&E-stained tumor slides of all patients were retrieved from our archives and re-evaluated independently in terms of budding according to the criteria of the ITBCC by two pathologists (CLS, BM) as described previously [25, 33]. Budding was reported as proposed: low budding $0-4$ buds (Bd1), intermediate budding 5-9 buds (Bd2), and high budding $\geq 10$ buds (Bd3) [29]. Only peritumoral budding at the invasive front was evaluated. For the budding-TIL groups, cases with intermediate $(\mathrm{Bd} 2)$ and high budding $(\mathrm{Bd} 3)$ were summarized as one "high budding group" as they had shown a trend to similar overall survival in our previous study [22].

The percentage of tumor-associated lymphatic infiltration was semiquantitatively estimated on the same H\&Estained slides, according to the ITWG methodology and as described before [30,31]. Referring to our previous studies, the cutoff for the low TILS group was set at $\leq 5 \%$ which resulted in four groups [22, 25]:

- Low budding/high TILs (i.e., Bd1 + TILs $>5 \%$ ).

- Low budding/low TILs (i.e., Bd1 + TILs $\leq 5 \%$ ).

- High budding/high TILs (i.e., Bd2 or Bd3 and TILs $>5 \%$ ).

- High budding/low TILs (i.e., $\mathrm{Bd} 2$ or $\mathrm{Bd} 3$ and TILs $\leq 5 \%$ ).

\section{PD-L1 immunohistochemistry}

PD-L1 immunohistochemistry was performed on whole tissue sections corresponding to those that had been used for budding and TIL assessment before. Four-micrometerthick slides were cut, and immunohistochemistry was performed with two different PD-L1 antibodies in order to obtain reproducible results:

- PD-L1 (clone 22C3 pharmDx, monoclonal mouse antihuman, dilution 1:50, Agilent, Santa Clara, CA, USA), which is the routinely used PD-L1 antibody in our laboratory.

- PD-L1 (clone QR1, monoclonal rabbit anti-human, dilution 1:150, Quartett, Berlin, Germany), which the FDA approval for pembrolizumab in AMMR CC is based on.
Immunostaining was performed using the fully automated Bond-III autostainer (Leica Biosystems, Wetzlar, Germany). Antigen retrieval was performed by heat-induced epitope retrieval (HIER) for $20 \mathrm{~min}$ at $100{ }^{\circ} \mathrm{C}$ with epitope retrieval solution 1 (ER1, citrate buffer, $\mathrm{pH}$ 6.0) for PD-L1, clone 22C3 and epitope retrieval solution 2 (ER2, EDTA-based puffer, $\mathrm{pH}$ 9.0) for PD-L1 clone QR1. The glass slides were incubated with each antibody for $15 \mathrm{~min}$ at room temperature. A Bond Polymer Red Refine Detection System (Leica Microsystems) was used for antibody detection and visualization, using Fast Red as chromogen. All slides were counterstained with hematoxylin for $7 \mathrm{~min}$, dehydrated in ascending grades of alcohol and covered by cover slips with Eukitt. Tonsil tissue served as on-slide positive control.

\section{PD-L1 assessment}

All PD-L1-stained tumor slides were assessed by one pathologist (CLS). The tumor proportion score (TPS) as well as the immune cell score (IC) was measured in percentages from 0 to 100. The TPS is defined as the percentage number of tumor cells with positive membranous staining of any intensity based on all tumor cells. The IC is defined as the percentage number of immune cells (granulocytes, dendritic cells, lymphocytes and macrophages) with positive cytoplasmic staining at any intensity based on all immune cells in the tumor area, including immune cells at the invasive front. Immune cells at the invasive front are included if they are within one field (in $20 \times$ objective magnification) with tumor occupying up to half of the field [34]. Combined positivity score (CPS) was also assessed (results not shown). Prior to the scoring and to learn the scoring method, the pathologist attended several web-based PD-L1 trainings and a password-secured PD-L1 training plattform (Qualitätssicherungs-Initiative Pathologie QuIP GmbH, Berlin, Germany, https://www.pdl1portal.eu/) to assess PD-L1 in line with currently valid scoring conventions.

PD-L1 immunohistochemistry was interpreted as follows: TPS and IC values of $\geq 1 \%$ were assigned as positive at any staining itensity. Score values of $0 \%$ or $<1 \%$ were assigned as negative [34].

Figure 1 shows histological example images of PD-L1 immunohistochemistry.

For interobserver reliability, a subset of 50, randomly chosen cases, was evaluated by a second pathologist (SW), trained in PD-L1 assessment. Both observers were blinded to each others' results.

\section{Statistics}

Statistical analyses were performed using the statistics program SPSS 21 (IBM Corp. Released 2012, IBM SPSS Statistics for Windows, Armonk, NY). Pearson's chi-squared test was used to test the association between different 


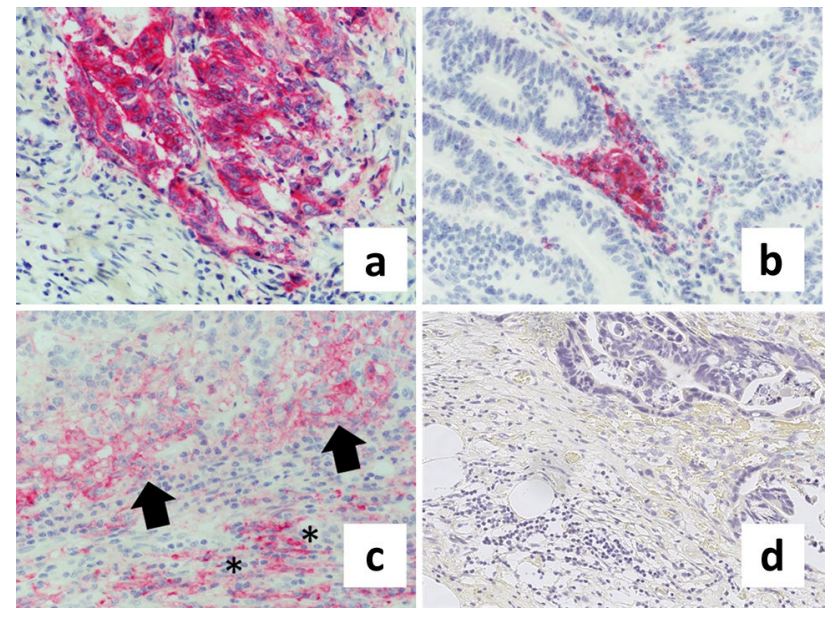

Fig. 1 Representative images of PD-L1 immunohistochemistry in colon cancer (CC). a TPS positivity, indicated by strong membranous PD-L1 staining of tumor cells in a patient with stage IV, mismatch repair deficient (dMMR) CC with low budding and high TILs (magnification $400 \times$ ). b IC positivity, defined as granular cytoplasmic positivity in immune cells in a patient with stage I, dMMR CC with low budding and low TILs (magnification $400 \times$ ). c Simultaneous membranous staining of tumor cells (arrows) and granular cytoplasmic staining of immune cells (asterisks) at the tumor invasive front in a patient with stage III, dMMR CC with low budding and high TILs (magnification $400 \times$ ). d PD-L1-negative tumor in a patient with stage IV, pMMR CC with high budding and low TILs (magnification $265 \times)$

parameters. Interobserver agreement was tested by Cohen's kappa. Univariate survival analyses for overall survival (OS) were carried out using the Kaplan-Meier method with logrank test. Multivariate survival analysis was performed using the Cox regression analysis. Hazard ratios and $95 \%$ confidence intervals (CIs) were used to determine effect size. $P$ values $<0.05$ were considered statistically significant.

\section{Results}

\section{Budding}

We found low budding in 266 (76.7\%), intermediate budding in $69(19.9 \%)$, and high budding in $12(3.5 \%)$ cases. Higher budding significantly correlated with higher $\mathrm{pT}(p=0.037)$ and $\mathrm{pN}$ stages $(p=0.033)$, higher TNM stage $(p=0.011)$, nonmucinous CC (not otherwise specified, NOS, $p=0.026$ ), L1 $(p=0.014)$, wild-type RAS ( $p=0.042)$, and MMR-proficient (pMMR) CC $(p=0.040)$.

Detailed results of the correlation analyses between tumor budding and clinicopathological features are available in Supplemental Table 1.

In Kaplan-Meier analysis, cases with low budding showed significantly longer disease-free survival (DFS) and overall survival (OS) than cases with intermediate budding $(p=0.022$ and $p=0.018)$. However, no significant difference was found between both groups and high budding.

\section{TILs}

One hundred seventy-seven cases $(51 \%)$ showed $\leq 5 \%$ TILs, and 170 cases $(49 \%)$ showed $>5 \%$ TILs. Higher amounts of TILs were significantly correlated with female gender $(p=0.002)$, less L1 $(p=0.001)$, and less V1 $(p=0.001)$ and correlated highly significant with lower $\mathrm{pT}(p<0.001)$, $\mathrm{pN}(p<0.001)$, and M stages $(p<0.001)$, as well as nonmucinous (NOS) CC $(p<0.001)$ and lower TNM stages $(p<0.001)$.

Detailed results of the correlation analyses between TILs and clinicopathological features are available in Supplemental Table 2.

In Kaplan-Meier analysis, cases with $>5 \%$ TILs showed significantly longer DFS $(p=0.015)$ and OS $(p=0.015)$ compared to cases with $\leq 5 \%$ TILs.

\section{Budding and TILs}

One hundred twenty-nine cases belonged to the low budding/ high TIL group (37.2\%), 128 (36.9\%) to the low budding/low TIL group, 42 (12.1\%) to the high budding/high TIL group, and 48 cases (13.8\%) to the high budding/low TIL group.

The combination of both parameters significantly correlated with gender, $\mathrm{pT}(p<0.001), \mathrm{pN}(p<0.001)$, M $(p<0.001)$, TNM stage $(p<0.001)$, mucinous versus NOS CC $(p<0.001), \mathrm{L} 1(p<0.001), \mathrm{V} 1(p=0.008)$, and MMR status $(p=0.041)$. No correlation was found with grading and KRAS.

Interobserver agreement between the two pathologists was substantial for budding $(\kappa=0.660, p<0.001)$ and fair for TILs $(\kappa=0.246, p=0.001)$.

Detailed results of the correlation analyses between the four budding/TIL groups and clinicopathological features are available as Supplemental Table 3.

In Kaplan-Meier analysis, DFS and OS were best for the low budding/high TIL group (mean DFS 93.439 months, 95\% CI 82.122-104.757 months; mean OS 93.862 months, 95\% CI 82.703-105.022 months) and worst for the high budding/low TIL group (mean DFS 47.927 months, 95\% CI 28.517-67.337 months; mean OS 55.369 months, 95\% CI 37.699-73.038 months). Kaplan-Meier analysis of OS for the four budding/TIL groups is shown in the supplemental figure. Differences between the four groups were statistically significant for DFS and OS between the low budding/high TIL group and the low budding/low TIL group ( $p=0.027$ for DFS and $p=0.023$ for OS) as well as between the low budding/high TIL group and the high budding/low TIL group ( $p=0.004$ for DFS and $p=0.005$ for OS) and showed a trend to longer OS for low budding/high TILs versus high budding/high TILs $(p=0.084)$. 
Table 2 Correlation between PD-L1 antibody, clone QR and the clinicopathological features

\begin{tabular}{|c|c|c|c|c|c|c|c|c|c|}
\hline \multirow[b]{3}{*}{ Feature } & \multicolumn{9}{|c|}{ PD-L1 IHC clone QR } \\
\hline & \multicolumn{3}{|l|}{ Overall } & \multicolumn{3}{|l|}{ TPS } & \multicolumn{3}{|c|}{ IC } \\
\hline & Positive $(n / \%)$ & Negative $(n / \%)$ & $p$ value & Positive $(n / \%)$ & Negative $(n / \%)$ & $p$ value & Positive $(n / \%)$ & Negative $(n / \%)$ & $p$ value \\
\hline $\begin{array}{l}\text { Gender } \\
\text { Male } \\
\text { Female }\end{array}$ & $\begin{array}{l}66(41.3) \\
97(52.4)\end{array}$ & $\begin{array}{l}94(58.8) \\
88(47.6)\end{array}$ & 0.025 & $\begin{array}{l}21(13.0) \\
40(21.5)\end{array}$ & $\begin{array}{l}140(87.0) \\
146(78.5)\end{array}$ & 0.027 & $\begin{array}{l}63(39.1) \\
94(50.5)\end{array}$ & $\begin{array}{l}98(60.9) \\
92(49.5)\end{array}$ & 0.022 \\
\hline $\begin{array}{l}\mathrm{pT} \\
1 \\
2 \\
3 \\
4\end{array}$ & $\begin{array}{l}9(40.9) \\
20(41.3) \\
104(50.7) \\
30(38.0)\end{array}$ & $\begin{array}{l}13(59.1) \\
19(48.7) \\
101(49.3) \\
49(62.0)\end{array}$ & 0.228 & $\begin{array}{l}3(13.0) \\
5(12.8) \\
40(19.4) \\
13(16.5)\end{array}$ & $\begin{array}{l}20(87.0) \\
34(87.2) \\
166(80.6) \\
66(83.5)\end{array}$ & 0.686 & $\begin{array}{l}9(39.1) \\
20(51.3) \\
99(48.1) \\
29(36.7)\end{array}$ & $\begin{array}{l}14(60.9) \\
19(48.7) \\
107(51.9) \\
50(63.3)\end{array}$ & 0.272 \\
\hline $\begin{array}{l}\mathrm{pN} \\
0 \\
1 \\
2\end{array}$ & $\begin{array}{l}108(54.5) \\
42(47.5) \\
17(25.4)\end{array}$ & $\begin{array}{l}90(45.5) \\
38(52.5) \\
50(74.6)\end{array}$ & $<0.001$ & $\begin{array}{l}36(18.2) \\
19(23.2) \\
6(9.0)\end{array}$ & $\begin{array}{l}162(81.8) \\
63(76.8) \\
61(91.0)\end{array}$ & 0.072 & $\begin{array}{l}105(53.0) \\
36(43.9) \\
16(23.9)\end{array}$ & $\begin{array}{l}93(47.0) \\
46(56.1) \\
51(76.1)\end{array}$ & $<0.001$ \\
\hline $\begin{array}{l}\mathrm{M} \\
0 \\
1\end{array}$ & $\begin{array}{l}154(53.1) \\
9(16.4)\end{array}$ & $\begin{array}{l}136(46.9) \\
46(83.6)\end{array}$ & $<0.001$ & $\begin{array}{l}56(19.2) \\
5(9.1)\end{array}$ & $\begin{array}{l}236(80.8) \\
50(90.9)\end{array}$ & 0.047 & $\begin{array}{l}149(51.0) \\
8(14.5)\end{array}$ & $\begin{array}{l}143(49.0) \\
47(85.5)\end{array}$ & $<0.001$ \\
\hline $\begin{array}{l}\text { Grading } \\
\text { Low grade } \\
\text { High grade }\end{array}$ & $\begin{array}{l}121(44.0) \\
42(60.0)\end{array}$ & $\begin{array}{l}154(56.0) \\
28(40.0)\end{array}$ & 0.012 & $\begin{array}{l}39(14.1) \\
22(31.4)\end{array}$ & $\begin{array}{l}238(95.9) \\
48(68.6)\end{array}$ & 0.001 & $\begin{array}{l}117(42.2) \\
40(57.1)\end{array}$ & $\begin{array}{l}160(57.8) \\
30(42.9)\end{array}$ & 0.018 \\
\hline $\begin{array}{l}\text { Mucinous } \\
\text { Yes } \\
\text { No (NOS) }\end{array}$ & $\begin{array}{l}7(29.2) \\
156(58.6)\end{array}$ & $\begin{array}{l}17(70.8) \\
165(51.4)\end{array}$ & 0.050 & $\begin{array}{l}0(0.0) \\
61(18.9)\end{array}$ & $\begin{array}{l}24(100.0) \\
262(81.1)\end{array}$ & 0.008 & $\begin{array}{l}7(29.2) \\
150(46.4)\end{array}$ & $\begin{array}{l}17(70.8) \\
173(53.6)\end{array}$ & 0.075 \\
\hline $\begin{array}{l}\text { TNM stage } \\
\text { I } \\
\text { II } \\
\text { III } \\
\text { IV }\end{array}$ & $\begin{array}{l}25(49.0) \\
83(59.3) \\
45(47.4) \\
10(16.9)\end{array}$ & $\begin{array}{l}26(51.0) \\
57(40.7) \\
50(52.6) \\
49(83.1)\end{array}$ & $<0.001$ & $\begin{array}{l}7(13.7) \\
30(21.4) \\
19(19.6) \\
5(8.5)\end{array}$ & $\begin{array}{l}44(86.3) \\
110(78.6) \\
78(80.4) \\
54(91.5)\end{array}$ & 0.133 & $\begin{array}{l}25(49.0) \\
80(57.1) \\
43(44.3) \\
9(15.3)\end{array}$ & $\begin{array}{l}26(51.0) \\
60(42.9) \\
54(55.7) \\
50(84.7)\end{array}$ & $<0.001$ \\
\hline $\begin{array}{l}\text { Localization } \\
\text { Right-sided } \\
\text { Left-sided }\end{array}$ & $\begin{array}{l}126(53.4) \\
37(33.9)\end{array}$ & $\begin{array}{l}110(46.6) \\
72(66.1)\end{array}$ & 0.001 & $\begin{array}{l}47(19.7) \\
14(12.8)\end{array}$ & $\begin{array}{l}191(80.3) \\
95(87.2)\end{array}$ & 0.076 & $\begin{array}{l}121(50.8) \\
36(33.0)\end{array}$ & $\begin{array}{l}117(49.2) \\
73(67.0)\end{array}$ & 0.001 \\
\hline $\begin{array}{l}\mathrm{L} \\
0 \\
1\end{array}$ & $\begin{array}{l}106(50.7) \\
57(41.99\end{array}$ & $\begin{array}{l}103(41.9) \\
79(58.1)\end{array}$ & 0.068 & $\begin{array}{l}35(16.6) \\
26(19.1)\end{array}$ & $\begin{array}{l}176(83.4) \\
110(80.9)\end{array}$ & 0.321 & $\begin{array}{l}104(49.3) \\
53(39.0)\end{array}$ & $\begin{array}{l}107(50.7) \\
83(61.0)\end{array}$ & 0.038 \\
\hline $\begin{array}{l}\mathrm{V} \\
0 \\
1\end{array}$ & $\begin{array}{l}138(50.7) \\
25(34.2)\end{array}$ & $\begin{array}{l}134(49.3) \\
48(65.8)\end{array}$ & 0.008 & $\begin{array}{l}49(17.9) \\
12(16.4)\end{array}$ & $\begin{array}{l}225(82.1) \\
61(83.6)\end{array}$ & 0.463 & $\begin{array}{l}132(48.2) \\
25(34.2)\end{array}$ & $\begin{array}{l}142(51.8) \\
48(65.8)\end{array}$ & 0.023 \\
\hline $\begin{array}{l}\text { KRAS } \\
\text { Wild type } \\
\text { Mutated }\end{array}$ & $\begin{array}{l}19(39.6) \\
16(36.4)\end{array}$ & $\begin{array}{l}29(60.4) \\
28(63.6)\end{array}$ & 0.459 & $\begin{array}{l}5(10.2) \\
4(9.1)\end{array}$ & $\begin{array}{l}44(89.8) \\
40(90.9)\end{array}$ & 0.569 & $\begin{array}{l}19(38.8) \\
15(34.1)\end{array}$ & $\begin{array}{l}30(61.2) \\
29(65.9)\end{array}$ & 0.401 \\
\hline $\begin{array}{l}\text { MMR } \\
\text { Proficient } \\
\text { Deficient }\end{array}$ & $\begin{array}{l}101(42.4) \\
48(65.8)\end{array}$ & $\begin{array}{l}137(57.6) \\
25(34.2)\end{array}$ & $<0.001$ & $\begin{array}{l}31(13.0) \\
23(31.5)\end{array}$ & $\begin{array}{l}208(87.0) \\
50(68.5)\end{array}$ & $<0.001$ & $\begin{array}{l}97(40.6) \\
47(64.4)\end{array}$ & $\begin{array}{l}142(59.4) \\
26(35.6)\end{array}$ & $<0.001$ \\
\hline $\begin{array}{l}\text { Budding } \\
\text { Low } \\
\text { Intermediate } \\
\text { High }\end{array}$ & $\begin{array}{l}130(49.2) \\
30(43.5) \\
3(25.0)\end{array}$ & $\begin{array}{l}134(50.8) \\
39(56.5) \\
9(75.0)\end{array}$ & 0.098 & $\begin{array}{l}52(19.5) \\
9(87.0) \\
0(0.0)\end{array}$ & $\begin{array}{l}214(80.5) \\
60(13.0) \\
12(100.0)\end{array}$ & 0.044 & $\begin{array}{l}126(47.4) \\
28(40.6) \\
3(25.0)\end{array}$ & $\begin{array}{l}140(52.6) \\
41(59.4) \\
9(75.0)\end{array}$ & 0.091 \\
\hline $\begin{array}{l}\text { TILs } \\
\leq 5 \% \\
>5 \%\end{array}$ & $\begin{array}{l}63(35.8) \\
100(59.2)\end{array}$ & $\begin{array}{l}113(64.2) \\
69(40.8)\end{array}$ & $<0.001$ & $\begin{array}{l}15(8.5) \\
46(27.1)\end{array}$ & $\begin{array}{l}162(91.5) \\
124(72.9)\end{array}$ & $<0.001$ & $\begin{array}{l}60(33.9) \\
97(57.1)\end{array}$ & $\begin{array}{l}117(66.1) \\
73(42.9)\end{array}$ & $<0.001$ \\
\hline
\end{tabular}

Statistically significant values are indicated in italics

$I H C$ immunohistochemistry, TPS tumor positivity score, IC immunocell score, TILs tumor-infiltrating lymphocytes, TNM tumor node metastasis, KRAS Kirsten rat sarkoma, $M M R$ mismatch repair 
Table 3 Correlation between PD-L1 antibody, clone 22C3 and the clinicopathological features

\begin{tabular}{|c|c|c|c|c|c|c|c|c|c|}
\hline \multirow[b]{3}{*}{ Feature } & \multicolumn{9}{|c|}{ PD-L1 IHC, clone 22C3 } \\
\hline & \multicolumn{3}{|l|}{ Overall } & \multicolumn{3}{|l|}{ TPS } & \multicolumn{3}{|l|}{ IC } \\
\hline & Positive $(n / \%)$ & Negative $(n / \%)$ & $p$ value & Positive $(n / \%)$ & Negative $(n / \%)$ & $p$ value & Positive $(n / \%)$ & Negative $(n / \%)$ & $p$ value \\
\hline $\begin{array}{l}\text { Gender } \\
\text { Male } \\
\text { Female }\end{array}$ & $\begin{array}{l}50(31.3) \\
55(29.6)\end{array}$ & $\begin{array}{l}110(68.8) \\
131(70.4)\end{array}$ & 0.412 & $\begin{array}{l}7(4.4) \\
18(9.7)\end{array}$ & $\begin{array}{l}153(95.6) \\
168(90.3)\end{array}$ & 0.044 & $\begin{array}{l}47(29.4) \\
49(26.3)\end{array}$ & $\begin{array}{l}113(70.6) \\
137(73.7)\end{array}$ & 0.306 \\
\hline $\begin{array}{l}\mathrm{pT} \\
1 \\
2 \\
3 \\
4\end{array}$ & $\begin{array}{l}2(8.7) \\
14(35.9) \\
76(36.9) \\
13(16.7)\end{array}$ & $\begin{array}{l}21(91.3) \\
25(64.1) \\
130(63.1) \\
65(83.3)\end{array}$ & 0.001 & $\begin{array}{l}1(4.3) \\
1(2.6) \\
16(7.8) \\
7(9.0)\end{array}$ & $\begin{array}{l}22(95.7) \\
38(97.4) \\
190(92.2) \\
71(91.0)\end{array}$ & 0.574 & $\begin{array}{l}2(8.7) \\
14(35.9) \\
71(34.5) \\
9(11.5)\end{array}$ & $\begin{array}{l}21(91.3) \\
25(64.1) \\
135(65.5) \\
69(88.5)\end{array}$ & $<0.001$ \\
\hline $\begin{array}{l}\mathrm{pN} \\
0 \\
1 \\
2\end{array}$ & $\begin{array}{l}72(36.4) \\
20(24.4) \\
13(19.7)\end{array}$ & $\begin{array}{l}126(63.6) \\
62(75.6) \\
53(80.3)\end{array}$ & 0.016 & $\begin{array}{l}16(8.1) \\
5(6.1) \\
4(6.1)\end{array}$ & $\begin{array}{l}182(91.9) \\
77(93.9) \\
62(93.9)\end{array}$ & 0.777 & $\begin{array}{l}67(33.8) \\
17(20.7) \\
12(18.2)\end{array}$ & $\begin{array}{l}131(66.2) \\
65(79.3) \\
54(81.8)\end{array}$ & 0.013 \\
\hline $\begin{array}{l}\mathrm{M} \\
0 \\
1\end{array}$ & $\begin{array}{l}101(34.6) \\
4(7.4)\end{array}$ & $\begin{array}{l}191(65.4) \\
50(92.6)\end{array}$ & $<0.001$ & $\begin{array}{l}23(7.9) \\
2(3.7)\end{array}$ & $\begin{array}{l}269(92.1) \\
52(96.3)\end{array}$ & 0.218 & $\begin{array}{l}93(31.8) \\
3(5.6)\end{array}$ & $\begin{array}{l}199(68.2) \\
51(94.4)\end{array}$ & $<0.001$ \\
\hline $\begin{array}{l}\text { Grading } \\
\text { Low grade } \\
\text { High grade }\end{array}$ & $\begin{array}{l}81(29.3) \\
24(34.3)\end{array}$ & $\begin{array}{l}195(70.7) \\
46(65.7)\end{array}$ & 0.254 & $\begin{array}{l}11(4.0) \\
14(20.0)\end{array}$ & $\begin{array}{l}265(96.0) \\
56(80.0)\end{array}$ & $<0.001$ & $\begin{array}{l}78(28.3) \\
18(25.7)\end{array}$ & $\begin{array}{l}198(71.7) \\
52(74.3)\end{array}$ & 0.396 \\
\hline $\begin{array}{l}\text { Mucinous } \\
\text { Yes } \\
\text { No (NOS) }\end{array}$ & $\begin{array}{l}6(25.0) \\
99(30.7)\end{array}$ & $\begin{array}{l}18(75.0) \\
223(69.3)\end{array}$ & 0.368 & $\begin{array}{l}0(0.0) \\
25(7.8)\end{array}$ & $\begin{array}{l}24(100.0) \\
297(92.2)\end{array}$ & 0.155 & $\begin{array}{l}6(25.0) \\
90(28.0)\end{array}$ & $\begin{array}{l}18(75.0) \\
232(72.0)\end{array}$ & 0.482 \\
\hline $\begin{array}{l}\text { TNM stage } \\
\text { I } \\
\text { II } \\
\text { III } \\
\text { IV }\end{array}$ & $\begin{array}{l}14(27.5) \\
58(41.4) \\
28(29.9) \\
5(8.6)\end{array}$ & $\begin{array}{l}37(72.5) \\
82(58.6) \\
69(91.1) \\
53(91.4)\end{array}$ & $<0.001$ & $\begin{array}{l}2(3.9) \\
14(10.0) \\
7(7.2) \\
2(3.4)\end{array}$ & $\begin{array}{l}49(96.1) \\
126(90.0) \\
90(92.8) \\
56(96.6)\end{array}$ & 0.299 & $\begin{array}{l}14(27.5) \\
53(37.9) \\
25(25.8) \\
4(6.9)\end{array}$ & $\begin{array}{l}37(72.5) \\
87(62.1) \\
72(74.2) \\
54(93.1)\end{array}$ & $<0.001$ \\
\hline $\begin{array}{l}\text { Localization } \\
\text { Right-sided } \\
\text { Left-sided }\end{array}$ & $\begin{array}{l}80(33.8) \\
25(22.9)\end{array}$ & $\begin{array}{l}157(66.2) \\
84(77.1)\end{array}$ & 0.027 & $\begin{array}{l}23(9.7) \\
2(1.8)\end{array}$ & $\begin{array}{l}214(90.3) \\
107(98.2)\end{array}$ & 0.005 & $\begin{array}{l}73(30.8) \\
23(23.1)\end{array}$ & $\begin{array}{l}164(69.2) \\
86(78.90)\end{array}$ & 0.039 \\
\hline $\begin{array}{l}\mathrm{L} \\
0 \\
1\end{array}$ & $\begin{array}{l}70(33.2) \\
35(25.9)\end{array}$ & $\begin{array}{l}141(66.8) \\
100(74.1)\end{array}$ & 0.094 & $\begin{array}{l}14(6.6) \\
11(8.1)\end{array}$ & $\begin{array}{l}197(93.4) \\
124(91.9)\end{array}$ & 0.371 & $\begin{array}{l}65(30.8) \\
31(23.0)\end{array}$ & $\begin{array}{l}146(69.2) \\
104(77.0)\end{array}$ & 0.071 \\
\hline $\begin{array}{l}\mathrm{V} \\
0 \\
1\end{array}$ & $\begin{array}{l}93(34.1) \\
12(16.4)\end{array}$ & $\begin{array}{l}180(65.9) \\
61(83.6)\end{array}$ & 0.002 & $\begin{array}{l}21(7.7) \\
4(5.5)\end{array}$ & $\begin{array}{l}252(92.3) \\
69(94.5)\end{array}$ & 0.361 & $\begin{array}{l}84(30.8) \\
12(16.4)\end{array}$ & $\begin{array}{l}189(69.2) \\
61(83.6)\end{array}$ & 0.009 \\
\hline $\begin{array}{l}\text { KRAS } \\
\text { Wild type } \\
\text { Mutated }\end{array}$ & $\begin{array}{l}10(20.4) \\
8(18.6)\end{array}$ & $\begin{array}{l}39(79.6) \\
35(81.4)\end{array}$ & 0.520 & $\begin{array}{l}4(8.2) \\
2(4.7)\end{array}$ & $\begin{array}{l}45(91.8) \\
41(95.3)\end{array}$ & 0.403 & $\begin{array}{l}7(14.3) \\
8(18.6)\end{array}$ & $\begin{array}{l}42(85.7) \\
35(81.4)\end{array}$ & 0.390 \\
\hline $\begin{array}{l}\text { MMR } \\
\text { Proficient } \\
\text { Deficient }\end{array}$ & $\begin{array}{l}63(26.4) \\
35(47.9)\end{array}$ & $\begin{array}{l}176(73.6) \\
38(52.1)\end{array}$ & $<0.001$ & $\begin{array}{l}9(3.8) \\
14(19.2)\end{array}$ & $\begin{array}{l}230(96.2) \\
59(80.8)\end{array}$ & $<0.001$ & $\begin{array}{l}97(40.6) \\
47(64.4)\end{array}$ & $\begin{array}{l}142(59.4) \\
26(35.6)\end{array}$ & $<0.001$ \\
\hline $\begin{array}{l}\text { Budding } \\
\text { Low } \\
\text { Intermediate } \\
\text { High }\end{array}$ & $\begin{array}{l}130(49.2) \\
30(43.5) \\
3(25.0)\end{array}$ & $\begin{array}{l}134(50.8) \\
39(56.5) \\
9(75.0)\end{array}$ & 0.098 & $\begin{array}{l}21(7.9) \\
3(4.3) \\
1(8.3)\end{array}$ & $\begin{array}{l}244(92.1) \\
66(95.7) \\
11(91.7)\end{array}$ & 0.490 & $\begin{array}{l}82(30.0) \\
12(17.4) \\
2(16.7)\end{array}$ & $\begin{array}{l}183(69.1) \\
57(82.6) \\
10(83.3)\end{array}$ & 0.022 \\
\hline $\begin{array}{l}\text { TILs } \\
\leq 5 \% \\
>5 \%\end{array}$ & $\begin{array}{l}63(35.8) \\
100(59.2)\end{array}$ & $\begin{array}{l}113(64.2) \\
69(40.8)\end{array}$ & $<0.001$ & $\begin{array}{l}15(8.5) \\
46(27.1)\end{array}$ & $\begin{array}{l}162(91.5) \\
124(92.9)\end{array}$ & $<0.001$ & $\begin{array}{l}60(33.9) \\
97(57.1)\end{array}$ & $\begin{array}{l}117(66.1) \\
73(42.9)\end{array}$ & $<0.001$ \\
\hline
\end{tabular}

Statistically significant values are indicated in italics

$I H C$ immunohistochemistry, TPS tumor positivity score, IC immune cell score, TILs tumor-infiltrating lymphocytes, TNM tumor node metastasis, KRAS Kirsten rat sarkoma, $M M R$ mismatch repair 


\section{PD-L1 immunohistochemistry}

Interobserver agreement between the two pathologists was substantial for PD-L1, clone QR $(\kappa=0.623, p<0.001)$ and fair for PD-L1, clone 22C3 $(\kappa=0.406, p=0.003)$. Staining was not assessable due to poor slide quality in two cases stained for clone QR and in one case stained for clone $22 \mathrm{C} 3$.

PD-L1 staining was completely negative in 182 cases (52.8\%) for clone QR and in 241 cases $(69.5 \%)$ for clone $22 \mathrm{C} 3$. Either TPS or IC was positive in 163 cases (47.2\%) for clone QR and in 105 cases (30.3\%) for clone 22C3. TPS was positive in 61 cases (17.6\%) for clone QR and in 25 cases (7.2\%) for clone 22C3. IC was positive in 157 cases (45.2\%) for clone QR and in 96 cases (27.7\%) for clone 22C3. The differences in overall staining positivity, TPS positivity, and IC positivity between both antibodies were statistically significant ( $p<0.001, p<0.001, p<0.001$, respectively).

\section{PD-L1 IHC, clone QR}

With clone QR, positivity in all three settings (overall, TPS, and IC) significantly correlated with TILs $>5 \%$ ( $p<0.001$ each), MMR deficiency ( $p<0.001$ each), high grade $(p=0.012, p=0.001$, and $p=0.018)$, female gender $(p=0.025, p=0.027$, and $p=0.022)$ and $\mathrm{M} 0(p<0.001$, $p=0.047$, and $p<0.001)$.

Additionally, overall PD-L1 positivity (TPS and/or IC) with clone QR significantly correlated with lower $\mathrm{pN}$ stages $(p<0.001)$, lower TNM stage $(p<0.001)$, right-sided colon cancer $(p=0.001)$, and less V1 $(p=0.008)$. Mucinous tumors were more often PD-L1 overall negative $(p=0.050)$, and cases with L1 showed a trend towards PD-L1 overall negativity $(p=0.068)$.

TPS positivity with clone QR additionally significantly correlated with low budding $(p=0.044)$, nonmucinous tumors (NOS, $p=0.008$ ) and showed a trend towards lower $\mathrm{pN}$ stages $(p=0.072)$ and right-sided location $(p=0.076)$.

IC positivity with clone QR-in addition to TILs $>5 \%$, dMMR, female gender, high grade, and M0 — significantly correlated with pN0 $(p<0.001)$ and lower TNM stage $(p<0.001)$. IC negativity was significantly more frequent in left-sided CC $(p=0.001)$, as well as tumors with L1 $(p=0.038)$ and V1 $(p=0.023)$ and showed a trend towards mucinous tumors $(p=0.075)$.

The results of the correlation analysis with PD-L1 antibody clone $\mathrm{QR}$ and the clinicopathological features are shown in Table 2.

\section{PD-L1 IHC, clone 22C3}

Concerning PD-L1 antibody clone 22C3, TILs $>5 \%$ and MMR deficiency were the only two clinicopathological features that were significantly associated with PD-L1 positivity in all three measures (overall, TPS, and IC; $p$ values $<0.001$ each for TILs $>5 \%$ and MMR deficiency). Left-sidedness of CC was the only feature that was associated with PD-L1-negative tumors in all three measures ( $p=0.027, p=0.005, p=0.039$, respectively).

Apart from left-sidedness, overall $22 \mathrm{C} 3$ negativity was significantly associated with higher pT stages $(p=0.001)$, higher pN stages $(p=0.016)$, higher TNM stages $(p<0.001)$, and V1 $(p=0.002)$. TPS was significantly more often negative in male patients $(p=0.044)$ and low-grade tumors $(p<0.001)$.

IC negativity correlated with higher $\mathrm{pT}(p<0.001)$ and $\mathrm{pN}$ stages $(p=0.013)$, higher TNM stage $(p<0.001)$, V1 $(p=0.009)$, and higher budding $(p=0.022)$.

The results of the correlation analysis with PD-L1 antibody clone, $22 \mathrm{C} 3$ and the clinicopathological features are shown in Table 3.

In Kaplan-Meier survival analysis, cases with PD-L1 IC positivity showed significantly longer OS compared to PDL1-negative cases with both antibodies ( $p=0.006$ for clone $\mathrm{QR}$ and $p=0.002$ for clone 22C3). The benefit in OS was even higher in the overall positivity cases (IC and/or TPS, $p=0.001$ for each antibody).

The results of the analysis between both antibodies and the four budding/TIL groups are shown in Table 4 (clone QR) and Table 5 (clone 22C3). With both antibodies, PD-L1-positive cases in both high TIL groups were significantly more frequent in the three PD-L1 categories (overall, IC, TPS) compared to both low TIL groups $(p<0.001$ each for clone QR and $p<0.001, p=0.002$ and $p<0.001$ for clone 22C3, respectively).

Concerning Kaplan-Meier analysis, with clone QR, the low bud/high TIL group was the only group that showed a significantly longer DFS and OS in the case of overall PD-L1 positivity compared to PD-L1-negative cases ( $p=0.045$ for DFS and $p=0.049$ for OS), whereas no differences were seen for TPS and IC as well as the other three budding/TIL groups. Kaplan-Meier analysis for OS for the low budding/ high TIL group is shown as Fig. 2.

Figure 3 shows representative histological images of PD-L1 immunohistochemistry (overall positive versus negative) for the low budding/high TIL group for each tumor stage (I-IV) and further separated into right-sided versus left-sided. As only 8 out of 129 patients in the low budding/high TIL group had stage IV CC, there was no case with PD-L1 positivity in the left-sided hemicolon.

With clone $22 \mathrm{C} 3$, a trend was seen towards better DFS and OS in low budding/high TIL cases with IC positivity ( $p=0.053$ for DFS and $p=0.067$ for OS). However, no significant differences were seen in overall positivity, TPS, and the other three budding/TIL groups.

\section{MMR-proficient cases}

MMR status was available for 312 cases. Among these, 239 (76.6) were pMMR and 73 (23.4\%) were dMMR. 
Table 4 Correlation between PD-L1-antibody, clone QR and the four budding/TIL groups

\begin{tabular}{|c|c|c|c|c|c|c|c|c|c|}
\hline \multirow[b]{3}{*}{$\begin{array}{l}\text { Budding/TIL } \\
\text { groups }\end{array}$} & \multicolumn{9}{|c|}{ PD-L1 IHC, clone QR } \\
\hline & \multicolumn{3}{|l|}{ Overall } & \multicolumn{3}{|l|}{ TPS } & \multicolumn{3}{|l|}{ IC } \\
\hline & Positive $(n / \%)$ & Negative $(n / \%)$ & $p$ value & Positive $(n / \%)$ & Negative $(n / \%)$ & $p$ value & Positive $(n / \%)$ & Negative $(n / \%)$ & $p$ value \\
\hline $\begin{array}{l}\text { Low buds/high } \\
\text { TILs } \\
(n=129)\end{array}$ & $76(59.4)$ & $52(40.6)$ & $<0.001$ & $39(30.2)$ & $90(69.8)$ & $<0.001$ & $74(57.4)$ & $55(42.6)$ & $<0.001$ \\
\hline $\begin{array}{l}\text { Low buds/low } \\
\text { TILs } \\
(n=128)\end{array}$ & $50(39.4)$ & $77(60.6)$ & & $10(7.8)$ & $118(92.2)$ & & $48(37.5)$ & $80(62.5)$ & \\
\hline $\begin{array}{l}\text { High buds/high } \\
\text { TILs } \\
(n=42)\end{array}$ & $24(57.1)$ & $18(42.9)$ & & $7(16.7)$ & $35(83.3)$ & & $23(54.8)$ & $19(45.2)$ & \\
\hline $\begin{array}{l}\text { High buds/low } \\
\text { TILs }(n=48)\end{array}$ & $13(27.1)$ & $35(72.9)$ & & $5(10.4)$ & $43(89.6)$ & & $12(25.0)$ & $36(75.0)$ & \\
\hline
\end{tabular}

Statistically significant values are indicated in italics

$I H C$ immunohistochemisty, TPS tumor positivity score, IC immune cell score, TILs tumor-infiltrating lymphocytes

The results of the correlation analysis between the four budding/TIL groups in pMMR cases and both PD-L1 antibodies are shown in Table 6 (clone QR) and Table 7 (clone $22 \mathrm{C} 3$ ). With both antibodies, cases in both high-TIL groups significantly correlated with PD-L1 overall and IC positivity $(p=0.008$ and $p=0.003$ for clone $\mathrm{QR}$ and $p=0.002$ each for clone 22C3). Both high-TIL groups showed longer OS in Kaplan-Meier analysis in PDL1-positive cases. However, the differences in OS between PD-L1 positive and negative cases in each of the four budding/TIL groups were not statistically significant.

Multivariate analysis revealed independent prognostic effects of PD-L1 positivity regarding $\mathrm{pT}(p<0.001)$, pN $(p<0.001)$, M $(p<0.001)$, TNM stage $(p<0.001)$, grading $(p=0.026)$, and V1 $(p<0.001)$ and showed a trend towards TILs $(p=0.076)$ and the budding/TIL combination $(p=0.084$, Table 8$)$.

\section{Discussion}

PD-L1 immunohistochemistry is mandatory for the decision proimmunooncogenic or contraimmunooncogenic treatment in tumors of different organs, for example, bladder cancer, lung cancer, or breast cancer [4, 35-38]. For CC, in June 2020, the FDA approved pembrolizumab (KEYTRUDA,

Table 5 Correlation between PD-L1-antibody, clone 22C3 and the four budding/TIL groups

\begin{tabular}{|c|c|c|c|c|c|c|c|c|c|}
\hline \multirow[b]{3}{*}{$\begin{array}{l}\text { Budding/TIL } \\
\text { groups }\end{array}$} & \multicolumn{9}{|c|}{ PD-L1 IHC, clone 22C3 } \\
\hline & \multicolumn{3}{|l|}{ Overall } & \multicolumn{3}{|l|}{ TPS } & \multicolumn{3}{|l|}{ IC } \\
\hline & Positive $(n / \%)$ & Negative $(n / \%)$ & $p$ value & Positive $(n / \%)$ & Negative $(n / \%)$ & $p$ value & Positive $(n / \%)$ & Negative $(n / \%)$ & $p$ value \\
\hline $\begin{array}{l}\text { Low buds/high } \\
\text { TILs } \\
(n=129)\end{array}$ & $60(46.5)$ & $69(43.5)$ & $<0.001$ & $18(14.0)$ & $111(86.0)$ & 0.002 & $54(41.9)$ & $75(58.1)$ & $<0.001$ \\
\hline $\begin{array}{l}\text { Low buds/low } \\
\text { TILs } \\
(n=128)\end{array}$ & $28(22.0)$ & $99(78.0)$ & & $3(2.4)$ & 124 (97.6) & & $26(20.5)$ & $101(79.5)$ & \\
\hline $\begin{array}{l}\text { High buds/high } \\
\text { TILs } \\
(n=42)\end{array}$ & $11(26.2)$ & $31(73.8)$ & & $3(7.1)$ & 39 (92.9) & & $10(23.8)$ & $32(76.2)$ & \\
\hline $\begin{array}{l}\text { High buds/low } \\
\text { TILs }(n=48)\end{array}$ & $6(12.5)$ & $42(87.5)$ & & $1(2.1)$ & 47 (97.9) & & $6(12.5)$ & $42(87.5)$ & \\
\hline
\end{tabular}

Statistically significant values are indicated in italics

IHC immunohistochemistry, TPS tumor positivity score, IC immune cell score, TILs tumor-infiltrating lymphocytes 
Fig. 2 Kaplan-Meier analysis showing overall survival (OS) for the low budding/high TIL group dependent on PD-L1 overall positivity (TPS and/ or IC positive) with clone QR. The difference between the PDL1-positive cases (blue) and the PD-L1-negative cases (green) was statistically significant $(p=0.049)$

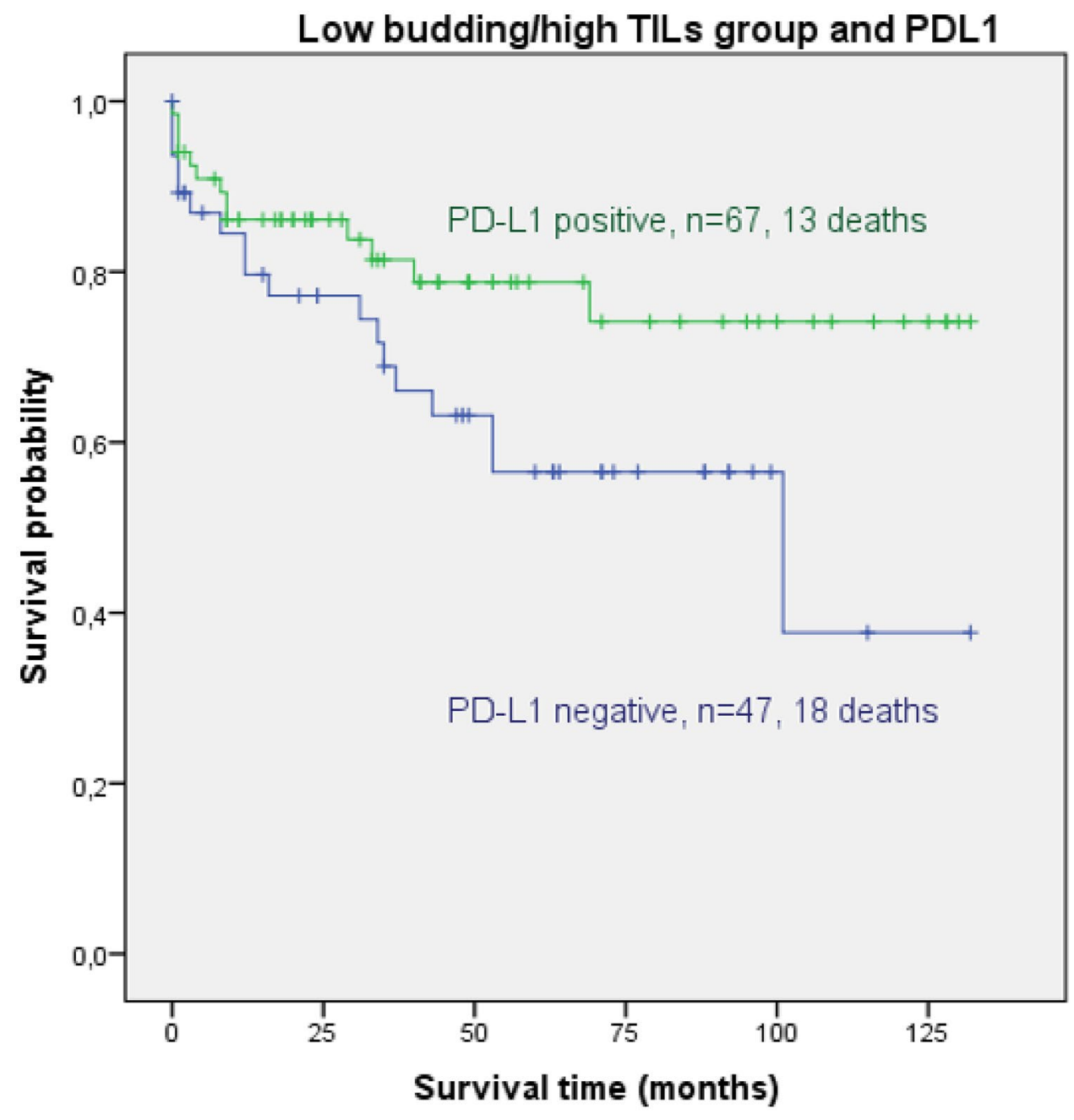

Merck Sharp Dohme) for first-line treatment of patients with unresectable or metastatic MSI-H or dMMR CRC, independent of PD-L1 immunohistochemisty [8].

However, the majority of CC patients belong to stages I to III and/or pMMR (in this cohort: dMMR in $23.4 \%$, only $1.9 \%$ thereof in stage IV versus $76.6 \%$ pMMR, stages I to IV). Therefore, there is a need to identify additional patients with CC who might benefit from immune therapy on the basis of their tumor biology and features of tumor microenvironment might be helpful for this issue.

The idea of analyzing budding (on the tumor side) and TILs (on the host side) as attacker-defender approach had was first described by Lugli et al. in 2009 [39].

In our previous studies, we were able to show that the combination of tumor budding and TILs as tumor-host antagonists is able to stratifiy patients with CC into prognostic subgroups with different OS. The parameter TILs proved to be more relevant regarding prognosis than the parameter budding. However, budding was also able to further stratify the low TIL cases into subgroups with different OS [22, 25]. Recently, we could further show that the budding/TIL combination is able to identify patients in stage II and III $\mathrm{CC}$ with and without benefit from adjuvant treatment [33].

Therefore, we aimed to analyze the interaction of PD-L1 immunohistochemistry with the budding/TIL combination to determine its potential value for the identification of additional candidates for immune therapy.

Studies focussing on PD-L1 immunohistochemistry in $\mathrm{CC}$ are very heterogenous in their scoring conventions, regarding, for example, amount of tissue for PD-L1 assessment (tissue micro array versus whole tumor slide), staining pattern (membranous versus cytoplasmatic), and cutoffs for PD-L1 positivity which make them difficult to compare. Elfishawy et al., for example, measured membranous staining only in tumor cells and stromal TILs and defined positivity as $>5 \%$ [40]. Wang et al. assessed PD-L1 in duplicate cores of $1 \mathrm{~mm}$ each on tissue micro arrays and also only evaluated membranous staining on tumor cells as well as immune cells, using stepwise cutoffs from $<1$ to $>10 \%$ [41].

Moreover, differences in staining quantities between different antibodies and staining platforms are well known, and 


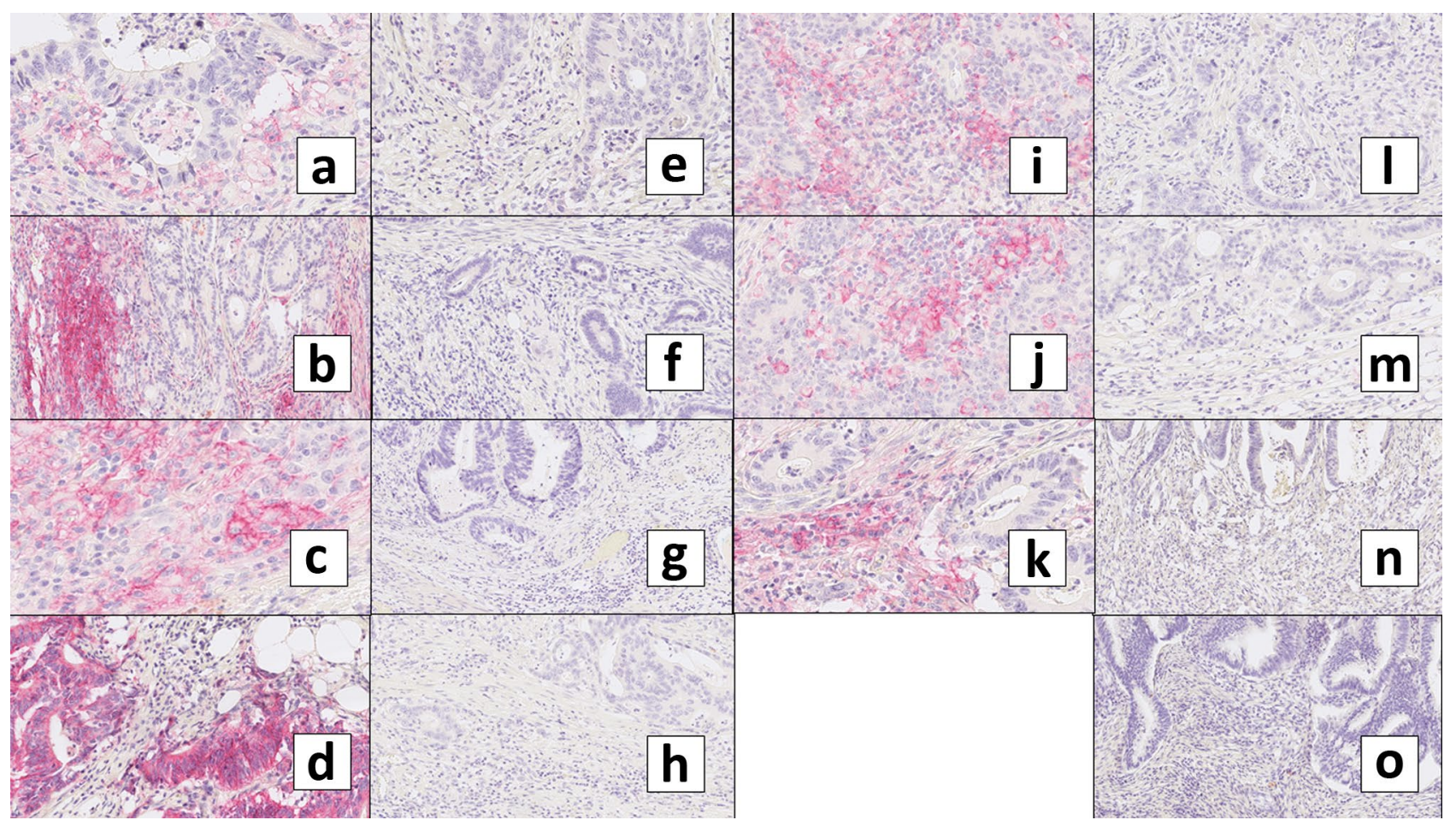

Fig. 3 Representative histological images of PD-L1 immunohistochemistry (overall positive versus negative) for the low budding/high TIL group for each tumor stage (I-IV) and further separated into right-sided (a-h) versus left-sided (i-o). As only 8 out of 129 patients in the low budding/high TIL group belonged to stage IV CC, there was no case with PD-L1 positivity in the left-sided hemicolon in

harmonization trials are conducted in order to provide recommendations for their use and different interpretations [42, 43]. When comparing the herein used two antibodies, with clone QR, more positive cases were found in PD-L1 overall positivity, TPS, and IC ( $47.2 \%, 17.6 \%$, and $45.2 \%$, respectively) compared to clone $22 \mathrm{C} 3(30.3 \%, 7.2 \%$, and $27.7 \%$, respectively). In our stage IV. a-d PD-L1 positive, right-sided (a stage I, b stage II, $\mathbf{c}$ stage III, d stage IV). e-h PD-L1 negative, right-sided (e stage I, f stage II, $\mathbf{g}$ stage III, $\mathbf{h}$ stage IV). i-k PD-L1 positive, left-sided (i stage I, $\mathbf{j}$ stage II, $\mathbf{k}$ stage III). l-o PD-L1 negative, left-sided (l stage I, $\mathbf{m}$ stage II, $\mathbf{n}$ stage III, $\mathbf{o}$ stage IV)

study, TILs $>5 \%$ and dMMR were the only two parameters that correlated reproducible and significantly with PD-L1 positivity in all three settings (overall, TPS, IC) with both antibodies.

Interestingly, overall and IC PD-L1 positivity with both antibodies were significantly linked to more favourable clinicopathological features like lower pT, pN, and $\mathrm{M}$ and less $\mathrm{L} 1$ and $\mathrm{V} 1$,

Table 6 Correlation between the four budding/TIL groups in pMMR cases and PD-L1 antibody, clone QR

\begin{tabular}{|c|c|c|c|c|c|c|c|c|c|}
\hline \multirow[b]{3}{*}{$\begin{array}{l}\text { Budding/TIL } \\
\text { groups }\end{array}$} & \multicolumn{9}{|c|}{ PD-L1 IHC, clone QR, MMR-proficient cases } \\
\hline & \multicolumn{3}{|l|}{ Overall } & \multicolumn{3}{|l|}{ TPS } & \multicolumn{3}{|l|}{ IC } \\
\hline & Positive $(n / \%)$ & Negative $(n / \%)$ & $p$ value & Positive $(n / \%)$ & Negative $(n / \%)$ & $p$ value & Positive $(n / \%)$ & Negative $(n / \%)$ & $p$ value \\
\hline $\begin{array}{l}\text { Low buds/high TILs } \\
(n=129)\end{array}$ & $44(53.0)$ & $39(47.0)$ & 0.008 & $16(19.3)$ & $67(80.7)$ & 0.181 & $44(53.0)$ & $39(47.0)$ & 0.003 \\
\hline $\begin{array}{l}\text { Low buds/low TILs } \\
(n=128)\end{array}$ & $29(34.1)$ & $56(65.9)$ & & $7(8.1)$ & $79(91.9)$ & & $27(31.4)$ & $59(68.6)$ & \\
\hline $\begin{array}{l}\text { High buds/high } \\
\text { TILs } \\
(n=42)\end{array}$ & $18(54.5)$ & $15(45.5)$ & & $4(12.1)$ & $29(87.9)$ & & $17(51.5)$ & $16(48.5)$ & \\
\hline $\begin{array}{l}\text { High buds/low TILs } \\
(n=48)\end{array}$ & $10(27.0)$ & $27(73.0)$ & & $4(10.8)$ & $33(89.2)$ & & $9(24.3)$ & $28(75.7)$ & \\
\hline
\end{tabular}

Statistically significant values are indicated in italics

$I H C$ immunohistochemistry, TPS tumor positivity score, IC immunecell score, TILs tumor-infiltrating lymphocytes 
Table 7 Correlation between the four budding/TIL groups in pMMR cases and PD-L1 antibody, clone 22C3

\begin{tabular}{|c|c|c|c|c|c|c|c|c|c|}
\hline \multirow[b]{3}{*}{ Budding/TIL groups } & \multicolumn{9}{|c|}{ PD-L1 IHC, clone 22C3, MMR-proficient cases } \\
\hline & \multicolumn{3}{|l|}{ Overall } & \multicolumn{3}{|l|}{ TPS } & \multicolumn{3}{|l|}{ IC } \\
\hline & Positive $(n / \%)$ & Negative $(n / \%)$ & $p$ value & Positive $(n / \%)$ & Negative $(n / \%)$ & $p$ value & Positive $(n / \%)$ & Negative $(n / \%)$ & $p$ value \\
\hline $\begin{array}{l}\text { Low buds/high TILs } \\
(n=129)\end{array}$ & $34(41.0)$ & $49(59.0)$ & 0.002 & $4(4.8)$ & $79(95.2)$ & 0.719 & $32(38.6)$ & $51(61.4)$ & 0.002 \\
\hline $\begin{array}{l}\text { Low buds/low TILs } \\
(n=128)\end{array}$ & $16(18.6)$ & $70(81.4)$ & & $2(2.3)$ & $84(97.7)$ & & $14(16.3)$ & $72(83.7)$ & \\
\hline $\begin{array}{l}\text { High buds/high TILs } \\
(n=42)\end{array}$ & $8(24.2)$ & $25(75.8)$ & & $2(6.1)$ & $31(93.9)$ & & $7(21.2)$ & $26(78.8)$ & \\
\hline $\begin{array}{l}\text { High buds/low TILs } \\
(n=48)\end{array}$ & $5(13.5)$ & $32(86.5)$ & & $1(2.7)$ & $36(97.3)$ & & $5(13.5)$ & $32(86.5)$ & \\
\hline
\end{tabular}

Statistically significant values are indicated in italics

$I H C$ immunohistochemistry, TPS tumor positivity score, IC immunecell score, TILs tumor-infiltrating lymphocytes

Table 8 Results of the multivariate analysis for clinical features, budding, TILs, and PD-L1 immunohistochemistry

\begin{tabular}{|c|c|c|c|}
\hline \multirow[b]{2}{*}{ Feature } & \multicolumn{3}{|c|}{ PD-L1 positivity } \\
\hline & & HR $(95 \% \mathrm{CI})$ & $p$ value \\
\hline pT & $\begin{array}{l}4 \\
1 \\
2 \\
3\end{array}$ & $\begin{array}{l}1.0 \\
0.510(0.239-1.102) \\
0.148(0.046-0.480) \\
0.481(0.320-0.722)\end{array}$ & $<0.001$ \\
\hline $\mathrm{pN}$ & $\begin{array}{l}2 \\
0 \\
1\end{array}$ & $\begin{array}{l}1.0 \\
0.400(0.256-0.626) \\
0.457(0.272-0.769)\end{array}$ & $<0.001$ \\
\hline M & $\begin{array}{l}1 \\
0\end{array}$ & $\begin{array}{l}1.0 \\
0.256(0.166-0.395)\end{array}$ & $<0.001$ \\
\hline TNM stage & $\begin{array}{l}\text { IV } \\
\text { I } \\
\text { II } \\
\text { III }\end{array}$ & $\begin{array}{l}1.0 \\
0.203(0.097-0.427) \\
0.229(0.137-0.382) \\
0.285(0.173-0.470)\end{array}$ & $<0.001$ \\
\hline Grading (WHO) & $\begin{array}{l}\text { High grade } \\
\text { Low grade }\end{array}$ & $\begin{array}{l}1.0 \\
0.586(0.374-0.920)\end{array}$ & 0.026 \\
\hline $\mathrm{V}$ & $\begin{array}{l}1 \\
0\end{array}$ & $\begin{array}{l}1.0 \\
0.448(0.300-0.667)\end{array}$ & $<0.001$ \\
\hline $\mathrm{L}$ & $\begin{array}{l}1 \\
0\end{array}$ & $\begin{array}{l}1.0 \\
0.744(0.511-1.085)\end{array}$ & 0.126 \\
\hline MMR status & $\begin{array}{l}\text { dMMR } \\
\text { pMMR }\end{array}$ & $\begin{array}{l}1.0 \\
1.472(0.834-2.597)\end{array}$ & 0.166 \\
\hline Budding & $\begin{array}{l}\text { high } \\
\text { low } \\
\text { intermediate }\end{array}$ & $\begin{array}{l}1.0 \\
1.051(0.331-3.339) \\
1.688(0.514-5.545)\end{array}$ & 0.111 \\
\hline TILs & $\begin{array}{l}>5 \% \\
\leq 5 \%\end{array}$ & $\begin{array}{l}1.0 \\
1.426(0.960-2.118)\end{array}$ & 0.076 \\
\hline $\begin{array}{l}\text { Score budding/ } \\
\text { TILs }\end{array}$ & $\begin{array}{l}\text { High buds/low } \\
\text { TILs } \\
\text { Low buds/high } \\
\text { TILs } \\
\text { Low buds/low } \\
\text { TILs } \\
\text { High buds/high } \\
\text { TILs }\end{array}$ & $\begin{array}{l}1.0 \\
0.545(0.310-0.960) \\
0.863(0.516-1.444) \\
1.016(0.504-2.050)\end{array}$ & 0.084 \\
\hline
\end{tabular}

Statistically significant values are indicated in italics

$H R$ hazard ratio, $C I$ confidence intervall, TILs tumor-infiltrating lymphocytes, TPS tumor positivity score, IC immune cell score, TNM tumor node metastasis, $M M R$ mismatch repair as well as lower tumor stage. PD-L1-positive cases (overall and IC) showed better OS. Wyss et al. found similar results in their study on 279 patients with CC. Stromal PD-L1 and PD-1 expressions were both associated with less aggressive tumor behavior and better OS and DFS [44]. Some studies showed a correlation between lower T stages, pN0, lower T stage, and PD-L1 positivity too; however, others did not [45-47]. Interestingly, PD-L1 positivity was also correlated with high grade and right-sidedness of $\mathrm{CC}$ which has also been shown by Wang et al. Kim et al. Elfishawy et al. and Lee et al. [40, 41, 48, 49].

Regarding the four budding/TIL groups, not surprisingly, PD-L1 positivity was statistically significantly more frequent in both high TIL groups. However, this was also the case when $\mathrm{dMMR}$ cases were excluded, allowing for the hypothesis that tumors with high TILs might respond to immune therapy even in the case of pMMR.

Interestingly, in our cohort, TILs did not correlate to MMR status but were almost equally distributed on dMMR and pMMR cases, which can probably be explained by our low cutoff for TIL stratification of 5\% and which is also in line with the study of Fuchs et al. on their large cohort of more than $1000 \mathrm{CC}$ patients, who also used the ITWG method for TIL assessment [32].

Most interestingly, the low budding/high TIL group was the only group that showed significantly better DFS and OS in the cases of PD-L1 overall positivity with clone QR (and a trend towards better DFS and OS with 22C3). This is interesting, as PD-L1 immunohistochemistry was able to further subdivide this group, which has already been shown to have the best OS of all four budding/TIL groups in our previous studies and is one of the two groups representing most CC cases [22].

\section{Conclusion}

As the outcome of patients with CC differs even in tumors with identical TNM stage, the focus of interest has switched to further characterization of tumor microenvironment in recent years. 
While elucidating the role of PD-L1 immunohistochemistry in $\mathrm{CC}$ with focus on its interaction with the budding and TIL combination, we were able to identify high grade, right-sidedness of CC, and tumors with TILs $>5 \%$, regardless of MMR status as parameters that might have potential to identify additional candidates that might benefit from immune therapy in CC. Additionally, patients with PD-L1 positivity in the low budding/high TIL group showed significantly better OS than PD-L1-negative cases. Further studies must show if patients in this group really have benefit from immune therapy. To the best of our knowledge, this is the first study analyzing the role of PD-L1 immunohistochemistry in the context of tumor budding and TILs as tumor-host antagonists. Further studies are necessary to elucidate if parameters of the tumor microenvironment can help in identifying patients with potential benefit from immune therapy in CRC, as there is currently no such option for the majority of CRC patients.

Supplementary information The online version contains supplementary material available at https://doi.org/10.1007/s00384-021-03985-9.

Acknowledgements The authors wish to thank Ingrid Hegenbarth, Stefanie Pirner, Manuela Stark, Rudolf Jung, and Michael Beer for excellent technical assistance.

Author contribution Conception and study design: Corinna LangSchwarz. Acquisition of data: Corinna Lang-Schwarz and Balint Melcher. Article drafting: Corinna Lang-Schwarz, William Sterlacci, and Simone Bertz. Analysis and interpretation of data: Corinna LangSchwarz and William Sterlacci. Critical revision of the article for important intellectual content: Corinna Lang-Schwarz, Simone Bertz, Arndt Hartmann, Theresa Dregelies, Klaus Lang-Schwarz, Michael Vieth, and William Sterlacci. Final approval of the submitted version: all authors.

Funding Open Access funding enabled and organized by Projekt DEAL. This study was supported by the Wissenschaftsförderung, Klinikum Bayreuth GmbH, grant number 50017, grant recipient: Dr. Corinna Lang-Schwarz.

Availability of data and material Data, as far as not anyway shown, are available on demand from the corresponding author via e-mail: Corinna.Lang-Schwarz@klinikum-bayreuth.de.

\section{Declarations}

Ethics approval The ethics commission of Friedrich-Alexander-University Erlangen-Nuremberg approved the study (study number 55_17 B).

Consent to participate All authors consented to participate.

Consent for publication All authors approved the final version of the manuscript for submission.

Competing interests The authors declare no competing interests.

Open Access This article is licensed under a Creative Commons Attribution 4.0 International License, which permits use, sharing, adaptation, distribution and reproduction in any medium or format, as long as you give appropriate credit to the original author(s) and the source, provide a link to the Creative Commons licence, and indicate if changes were made. The images or other third party material in this article are included in the article's Creative Commons licence, unless indicated otherwise in a credit line to the material. If material is not included in the article's Creative Commons licence and your intended use is not permitted by statutory regulation or exceeds the permitted use, you will need to obtain permission directly from the copyright holder. To view a copy of this licence, visit http://creativecommons.org/licenses/by/4.0/.

\section{References}

1. Global Cancer Observatory (2020) Cancer Today. Lyon, France: International Agency for Research on Cancer. Available from: https://gco.iarc.fr/today. Accessed April 22, 2021

2. Nagtegaal ID, Arends MJ, Odze RD, Lam AK (2019) Tumours of the colon and rectum. In: Lokuhetty D, White VA, Watanabe R, Cree IA (ed). WHO Classification of Tumours. Digestive system tumours. Lyon: International Agency for Research on Cancer pp 177-187

3. Schachter J, Ribas A, Long GV et al (2017) Pembrolizumab versus ipilimumab for advanced melanoma: final overall survival results of a multicentre, randomised, open-label phase 3 study (KEYNOTE-006). Lancet 390(10105):1853-1862. https://doi.org/10.1016/S01406736(17)31601-X

4. Reck M, Rodríguez-Abreu D, Robinson AG et al (2016) KEYNOTE-024 Investigators. Pembrolizumab versus chemotherapy for PD-L1positive nonsmall-cell lung cancer. N Engl J Med 375(19):1823-1833. https://doi.org/10.1056/NEJMoa1606774

5. Schmid P, Adams S, Rugo HS, Schneeweiss A, Barrios CH, Iwata H, Dieras V, Hegg R, Im S, Shaw Wright G, Henschel V, Molinero L, Chui SY, Funke R, Husain A, Winer EP, Loi S, Emens LA (2018) Atezolizumab and nab-paclitaxel in advanced triple-negative breast cancer. N Engl J Med 379(22):2108-2121. https://doi.org/10.1056/NEJMoa1809615

6. Schmid P, Rugo HS, Adams S, Schneeweiss A, Barrios CH, Iwata H, Diéras V, Henschel V, Molinero L, Chui SY, Maiya V, Husain A, Winer EP, Loi S, Emens LA, IMpassion130 Investigators (2020) Atezolizumab plus nab-paclitaxel as first-line treatment for unresectable, locally advanced or metastatic triplenegative breast cancer (IMpassion130): updated efficacy results from a randomised, double-blind, placebo-controlled, phase 3 trial Lancet Oncol 2114459 https://doi.org/10.1016/S14702045(19)30689-8

7. André T, Shiu KK, Kim TW et al (2020) Pembrolizumab in microsatellite-instability-high advanced colorectal cancer. N Engl J Med 383(23):2207-2218. https://doi.org/10.1056/NEJMoa2017699

8. Food and Drug Administration (2020) Available from: https:// www.accessdata.fda.gov/scripts/cder/daf/index.cfm?event $=$ overview.process\&ApplNo=125514. accessed 04/25/2021

9. Koopman M, Kortman GAM, Mekenkamp L et al (2009) Deficient mismatch repair system in patients with sporadic advanced colorectal cancer. Br J Cancer 27;100(2):266-73. https://doi.org/ 10.1038/sj.bjc.6604867

10. Latham A, Srinivasan P, Kemel Y et al (2019) Microsatellite instability is associated with the presence of Lynch syndrome pancancer. J Clin Oncol 37(4):286-295. https://doi.org/10.1200/JCO. 18.00283

11. Bonneville R, Krook MA, Kautto EA et al (2017) Landscape of microsatellite instability across 39 cancer types. JCO Precis Oncol 2017:PO.17.00073. https://doi.org/10.1200/PO.17.00073 
12. Le DT, Durham JN, Smith KN et al (2017) Mismatch repair deficiency predicts response of solid tumors to PD-1 blockade. Science 357:409-413. https://doi.org/10.1126/science.aan6733

13. Venderbosch S, Nagtegaal ID, Maughan TS et al (2014) Mismatch repair status and BRAF mutation status in metastatic colorectal cancer patients: a pooled analysis of the CAIRO, CAIRO2, COIN, and FOCUS studies. Clin Cancer Res 20:5322-5330. https://doi. org/10.1158/1078-0432.CCR-14-0332

14. Lugli A, Karamitopoulou E, Zlobec I (2012) Tumour budding: a promising parameter in colorectal cancer. Br J Cancer 106:17131717. https://doi.org/10.1038/bjc.2012.127

15. Van Wyk HC, Park J, Roxburgh C, Horgan P, Foulis A, McMillan DC (2015) The role of tumour budding in predicting survival in patients with primary operable colorectal cancer: a systematic review. Cancer Treat Rev 41:151-159. https://doi.org/10.1016/j. ctrv.2014.12.007

16. Rogers AC, Winter DC, Heeney A, Gibbons D, Lugli A, Puppa G, Sheahan K (2016) Systematic review and meta-analysis of the impact of tumour budding in colorectal cancer. Br J Cancer 115:831-840. https://doi.org/10.1038/bjc.2016.274

17. Ueno H, Murphy J, Jass JR, Mochizuki H, Talbot IC (2002) Tumour budding as an index to estimate the potential of aggressiveness in rectal cancer. Histopathology 40:127-132. https://doi. org/10.1046/j.1365-2559.2002.01324.X

18. Ueno H, Mochizuki H, Hashiguchi Y, Hatsuse K, Fujimoto H, Hase K (2004) Predictors of extrahepatic recurrence after resection of colorectal liver metastases. Br J Surg 91:327-333. https:// doi.org/10.1002/bjs.4429

19. Ueno H, Mochizuki H, Hashiguchi Y et al (2004) Risk factors for an adverse outcome in early invasive colorectal carcinoma. Gastroenterology 127:385-394. https://doi.org/10.1053/j.gastro. 2004.04.022

20. Kazama S, Watanabe T, Ajioka Y, Kanazawa T, Nagawa H (2006) Tumour budding at the deepest invasive margin correlates with lymph node metastasis in submucosal colorectal cancer detected by anticytokeratin antibody CAM5.2. Br J Cancer 94:293-298. https://doi.org/10.1038/sj.bjc.6602927

21. Mitrovic B, Schaeffer DF, Riddell RH, Kirsch R (2012) Tumor budding in colorectal carcinoma: time to take notice. Mod Pathol 25:1315-1325. https://doi.org/10.1038/modpathol.2012.94

22. Lang-Schwarz C, Melcher B, Haumaier F, Lang-Schwarz K, Rupprecht T, Vieth M, Sterlacci W (2018) Budding and tumorinfiltrating lymphocytes - combination of both parameters predicts survival in colorectal cancer and leads to new prognostic subgroups. Hum Pathol 79:160-167. https://doi.org/10.1016/j. humpath.2018.05.010

23. Grigore AD, Jolly MK, Jia D, Farach-Carson MC, Levine H (2016) Tumor budding: the name is EMT. Partial EMT J Clin Med 5:51. https://doi.org/10.3390/jcm5050051

24. Zlobec I, Lugli A (2010) Epithelial mesenchymal transition and tumor budding in aggressive colorectal cancer: tumor budding as oncotarget. Oncotarget 1:651-661. https://doi.org/10.18632/oncotarget.199

25. Lang-Schwarz C, Melcher B, Haumaier F, Schneider-Fuchs A, Lang-Schwarz K, Krugmann J, Vieth M, Sterlacci W (2019) Budding, tumor-infiltrating lymphocytes, gland formation: scoring leads to new prognostic groups in World Health Organization low-grade colorectal cancer with impact on survival. Hum Pathol 89:81-89. https://doi.org/10.1016/j.humpath.2019.04.006

26. Compton CC (2016) Pathology and prognostic determinants of colorectal cancer. In:Savarese DM (ed). UpToDate; Waltham, MA, USA:2016

27. Mei Z, Liu Y, Liu C, Cui A, Liang Z, Wang G, Peng H, Cui L, Li $\mathrm{C}$ (2014) Tumour-infiltrating inflammation and prognosis in colorectal cancer: systematic review and meta-analysis. Br J Cancer 110:1595-1605. https://doi.org/10.1038/bjc.2014.46
28. Huh JW, Lee JH, Kim HR (2012) Prognostic significance of tumor-infiltrating lymphocytes for patients with colorectal cancer. Arch Surg 4(147):366-372. https://doi.org/10.1001/archsurg. 2012.35

29. Lugli A, Kirsch R, Ajioka Y et al (2016) Recommendations for reporting tumor budding in colorectal cancer based on the International Tumor Budding Consensus Conference (ITBCC) 2016. Mod Pathol 30(9):1299-1311. https://doi.org/10.1038/modpathol. 2017.46

30. Salgado R, Denkert C, Demaria S et al (2015) The evaluation of tumor-infiltrating lymphocytes (TILs) in breast cancer: recommendations by an International TILs Working Group 2015. Ann Oncol 26:259-271. https://doi.org/10.1093/annonc/mdu450

31. Hendry S, Salgado R, Gevaert T et al (2017) Assessing tumorinfiltrating lymphocytes in solid tumors: a practical review for pathologists and proposal for a standardized method from the International Immuno-Oncology Biomarkers Working Group: Part 2: TILs in Melanoma, Gastrointestinal Tract Carcinomas, Non-Small Cell Lung Carcinoma and Mesothelioma, Endometrial and Ovarian Carcinomas, Squamous Cell Carcinoma of the Head and Neck, Genitourinary Carcinomas, and Primary Brain Tumors. Adv Anat Pathol 24(6):311-335. https://doi.org/10.1097/ PAP.0000000000000162

32. Fuchs TL, Sioson L, Sheen A, Jafari-Nejad K, Renaud CJ, Andrici J, Ahadi M, Chou A, Gill AJ (2020) Assessment of tumor-infiltrating lymphocytes using International TILs Working Group (ITWG) system is a strong predictor of overall survival in colorectal carcinoma. A Study of 1034 Patients. Am J Surg Pathol 44(4):536-544. https:// doi.org/10.1097/PAS.0000000000001409

33. Lang-Schwarz C, Melcher B, Dregelies T, Norouzzadeh Z, RundKüffner S, Lang-Schwarz K, Vieth M, Sterlacci W (2021) Adjuvant chemotherapy in stage ii and iii colon cancer: the role of the "budding and TILs-(tumor infiltrating lymphocytes) combination" as tumor-host antagonists. Int J Colorectal Dis. Published online ahead of print: 20.March 2021. https://doi.org/10.1007/ s00384-021-03896-9

34. Schildhaus HU (2018) Predictive value of PD-L1 diagnostics. Pathologe 39(6):498-519. https://doi.org/10.1007/ s00292-018-0507-x

35. Balar AV, Galsky MD, Rosenberg JE et al (2017) Atezolizumab as first-line treatment in cisplatin-ineligible patients with locally advanced and metastatic urothelial carcinoma: a single-arm, multicentre, phase 2 trial. Lancet 7;389(10064):67-76. https://doi.org/ 10.1016/S0140-6736(16)32455-2

36. Balar AV, Castellano D, O'Donnell PH et al (2017) First-line pembrolizumab in cisplatin-ineligible patients with locally advanced and unresectable or metastatic urothelial cancer (KEYNOTE-052): a multicentre, single-arm, phase 2 study. Lancet Oncol 18(11):14831492. https://doi.org/10.1016/S1470-2045(17)30616-2

37. Gourd E (2018) EMA restricts use of Keytruda and Tecentriq in bladder cancer. Lancet Oncol 19(7):e341. https://doi.org/10.1016/ S1470-2045(18)30433-9

38. Cortes J, Cescon DW, Rugo HS et al (2020) Pembrolizumab plus chemotherapy versus placebo plus chemotherapy for previously untreated locally recurrent inoperable or metastatic triple-negative breast cancer (KEYNOTE-355): a randomised, placebo-controlled, double-blind, phase 3 clinical trial. Lancet 396(10265):1817-1828. https://doi.org/10.1016/S0140-6736(20)32531-9

39. Lugli A, Karamitopoulou E, Panayiotides I et al (2009) CD8+ lymphocytes/ tumour-budding index: an independent prognostic factor representing a "pro-/anti-tumour" approach to rumour host interaction in colorectal cancer. Br J Cancer 101:1382-1392. https://doi.org/10.1038/sj.bjc.6605318

40. Elfishawy M, Abd-ELaziz SA, Hegazy A, El-yasergy DF (2020) Immunohistochemical expression of programmed death ligand-1 
(pdl-1) in colorectal carcinoma and its correlation with stromal tumor infiltrating lymphocytes. Asian Pac J Cancer Prev 21(1):225-232. https://doi.org/10.31557/APJCP.2020.21.1.225

41. Wang L, Ren F, Wang Q et al (2016) Significance of programmed death ligand 1 (pd-11) immunohistochemical expression in colorectal cancer. Mol Diagn Ther 20(2):175-181. https://doi.org/10. 1007/s40291-016-0188-1

42. Adam J, Le Stang N, Rouquette I et al (2018) Multicenter harmonization study for PD-L1 IHC testing in non-small-cell lung cancer. Ann Oncol 29(4):953-958. https://doi.org/10.1093/annonc/ mdy014

43. Scheel AH, Dietel M, Heukamp LC et al (2016) Harmonized PD-L1 immunohistochemistry for pulmonary squamous-cell and adenocarcinomas. Mod Pathol 29(10):1165-1172. https://doi.org/ 10.1038/modpathol.2016.117

44. Wyss J, Dislich B, Koelzer VH et al (2019) Stromal PD-1/PD-L1 expression predicts outcome in colon cancer patients. Clin Colorectal Cancer 18(1):e20-e38. https://doi.org/10.1016/j.clcc.2018. 09.007

45. Droeser RA, Hirt C, Viehl CT et al (2013) Clinical impact of programmed cell death ligand 1 expression in colorectal cancer. Eur $\mathrm{J}$ Cancer 49(9):2233-2242. https://doi.org/10.1016/j.ejca.2013.02. 015
46. Rosenbaum MW, Bledsoe JR, Morales-Oyarvide V et al (2016) PD-L1 expression in colorectal cancer is associated with microsatellite instability, BRAF mutation, medullary morphology and cytotoxic tumor-infiltrating lymphocytes. Mod Pathol 29(9):11041112. https://doi.org/10.1038/modpathol.2016.95

47. Masugi Y, Nishihara R, Yang J et al (2016) Tumour CD274 (PDL1) expression and T cells in colorectal cancer. Gut 66(8):14631473. https://doi.org/10.1136/gutjnl-2016-311421

48. Kim JH, Park HE, Cho NY et al (2016) Characterisation of PDL1-positive subsets of microsatellite-unstable colorectal cancers Br J Cancer 115(4):490-6. https://doi.org/10.1038/bjc.2016.211

49. Lee LH, Cavalcanti MS, Segal NH et al (2016) Patterns and prognostic relevance of PD-1 and PD-L1 expression in colorectal carcinoma. Mod Pathol 29(11):1433-1442. https://doi.org/10.1038/ modpathol.2016.139

Publisher's Note Springer Nature remains neutral with regard to jurisdictional claims in published maps and institutional affiliations. 